\title{
MHD simulations of supernova driven ISM turbulence
}

\section{Oliver Gressel ${ }^{1}$ and Udo Ziegler}

MHD group, Astrophysikalisches Institut Potsdam An der Sternwarte 16, 14482 Potsdam, Germany

${ }^{1}$ email: ogressel@aip.de

\begin{abstract}
Large-scale magnetic fields, that can be observed in numerous galaxies, are most likely the outcome of a dynamic process, a so-called dynamo. The favoured mechanisms for driving such a process in the ISM are supernovae(SNe) and/or magneto-rotational instability(MRI). In this work we simulate the dynamic evolution of the turbulent ISM utilising a three-dimensional MHD model.
\end{abstract}

Keywords. turbulence, ISM: kinematics and dynamics, magnetic fields, methods: numerical

Model description: Adopting the early models of Korpi et al. (1999) our spatial domain covers a box of $0.5^{2} \times 2.0 \mathrm{kpc}^{3}$ at a resolution of $\sim 4 \mathrm{pc}$. The adiabatic equation of state is supplemented by a parameterised heating- and cooling-function allowing for thermal instability(TI). The update due to heating and cooling is implemented implicitly using a Patankar-type discretisation. Turbulence is driven by SNe which are modelled as local injections of thermal energy. SN-rates are adopted for typical cited values. We make a distinction between typeI and typeII SNe. Latter are clustered by the (artificial) constraint that the density at the explosion site be above average, former are spatially uncorrelated. The initial setup includes a differentially rotating background (with shearing $\mathrm{BCs}$ in radial direction) as well as vertical stratification. The initial density- and pressureprofiles are numerically integrated ensuring hydrostatic equilibrium with respect to the equation of state given by the radiative equilibrium. Including z-dependent heating rates this leads to a considerable deviation from usual isothermal initial models.

Preliminary Results: The amplification of the turbulent magnetic field is found to be independent of seed field amplitudes. Typical e-folding times for the magnetic energy are $\sim 1 / 10$ of the galactic period. Obtained velocity dispersions are $\sim 25 \mathrm{~km} \mathrm{~s}^{-1}$ and thus at the upper limit of observed values.

Implications: The primary focus of this work is on the galactic dynamo and the generation of large-scale magnetic fields. As a secondary target we are interested in general properties of the ISM that are of importance for star formation(SF). To date only few simulations of gravo-turbulent SF do include magnetic fields or SN-feedback. Our model, however, lacks self-gravity. Peak densities are $\sim 10^{2} \mathrm{~cm}^{-3}$, well below typical values for molecular clouds.

\section{Acknowledgements}

This work was supported by DFG under grant ZI 717/2-2.

\section{Reference}

Korpi, M., Brandenburg, A., Shukurov, A., Tuominen, I. \& Nordlund, Å. 1999, ApJ 514, L99 